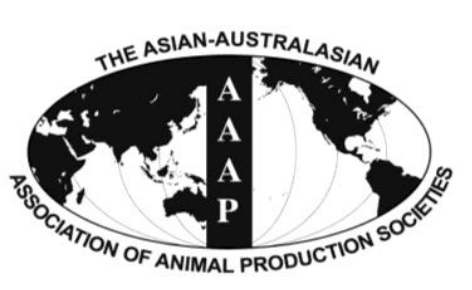

Open Access

Asian Australas. J. Anim. Sci.

Vol. 27, No. 12 : 1695-1704 December 2014

http://dx.doi.org/10.5713/ajas.2014.14335

www.ajas.info

pISSN 1011-2367 elSSN 1976-5517

\title{
Maternal Low-protein Diet Alters Ovarian Expression of Folliculogenic and Steroidogenic Genes and Their Regulatory MicroRNAs in Neonatal Piglets
}

\author{
Shiyan Sui ${ }^{1,2}$, Yimin $\mathrm{Jia}^{1}$, Bin $\mathrm{He}^{1}$, Runsheng $\mathrm{Li}^{1}$, Xian $\mathrm{Li}^{1}$, Demin Cai ${ }^{1}$, Haogang Song ${ }^{1}$, \\ Rongkui Zhang ${ }^{3}$, and Ruqian Zhao ${ }^{1, *}$ \\ ${ }^{1}$ Key Laboratory of Animal Physiology and Biochemistry, Ministry of Agriculture, \\ Nanjing Agricultural University, Nanjing 210095, China
}

\begin{abstract}
Maternal malnutrition during pregnancy may give rise to female offspring with disrupted ovary functions in adult age. Neonatal ovary development predisposes adult ovary function, yet the effect of maternal nutrition on the neonatal ovary has not been described. Therefore, here we show the impact of maternal protein restriction on the expression of folliculogenic and steroidogenic genes, their regulatory microRNAs and promoter DNA methylation in the ovary of neonatal piglets. Sows were fed either standardprotein (SP, 15\% crude protein) or low-protein (LP, 7.5\% crude protein) diets throughout gestation. Female piglets born to LP sows showed significantly decreased ovary weight relative to body weight $(\mathrm{p}<0.05)$ at birth, which was accompanied with an increased serum estradiol level $(\mathrm{p}<0.05)$. The LP piglets demonstrated higher ratio of bcl-2 associated X protein/B cell lymphoma/leukemia-2 mRNA $(p<0.01)$, which was associated with up-regulated mRNA expression of bone morphogenic protein $4(B M P 4)(p<0.05)$ and proliferating cell nuclear antigen (PCNA) ( $\mathrm{p}<0.05)$. The steroidogenic gene, cytochrome P450 aromatase (CYP19A1) was significantly downregulated $(\mathrm{p}<0.05)$ in LP piglets. The alterations in ovarian gene expression were associated with a significant down-regulation of follicle-stimulating hormone receptor mRNA expression $(\mathrm{p}<0.05)$ in LP piglets. Moreover, three microRNAs, including miR-423-5p targeting both CYP19A1 and PCNA, miR-378 targeting CYP19A1 and miR-210 targeting BMP4, were significantly down-regulated $(\mathrm{p}<0.05)$ in the ovary of LP piglets. These results suggest that microRNAs are involved in mediating the effect of maternal protein restriction on ovarian function through regulating the expression of folliculogenic and steroidogenic genes in newborn piglets. (Key Words: Folliculogenesis, Steroidogenesis, Maternal Dietary Protein, MicroRNA, Ovary, Neonatal Piglet)
\end{abstract}

\section{INTRODUCTION}

Maternal nutrition plays an important role in the programming of offspring health and disease (Godfrey et al., 2010). Studies in animals and human have shown that prenatal nutrition influences ovarian function and the pubertal timing in offspring (Sloboda et al., 2009; Sloboda

\footnotetext{
* Corresponding Author: Ruqian Zhao. Tel: +86-2584395047, Fax: +86-2584398669, E-mail: zhao.ruqian@ gmail.com

2 The Department of Agriculture and Biological Sciences, Dali University of Yunnan Province, Dali 671003, China.

${ }^{3}$ Shanghai Farm of Bright Food (Group) Co., Ltd, Dafeng 224100, China.

Submitted May 8, 2014; Revised Jul. 25, 2014; Accepted Aug. 23, 2014
}

et al., 2011). Maternal malnutrition during pregnancy disrupts ovarian folliculogenesis (Bernal et al., 2010) and reduces ovulation rate in adult offspring of rodents (Meikle and Westberg, 2001), sheep (Rae et al., 2002) and human (Ibáñez et al., 2002).

In mammals, primordial follicles begin to form before or during the first few days after birth (Faria et al., 2010). Then the primordial follicles transit into primary follicles and initiate folliculogenesis (Faria et al., 2010). The number of primordial follicles in the ovary is finite and represents complete reproductive potential for the individual (da Silva Faria et al., 2008). Abnormal folliculogenesis in neonatal life leads to long-term disruption of ovarian function in adult life (Fortune et al., 2000; Durlej et al., 2011). 
However, studies concerning the effect of maternal malnutrition on offspring ovarian function are primarily focused on adults; little is known how maternal low-protein (LP) diet during pregnancy alters neonatal ovarian development in offspring.

The development of early follicles is regulated by numerous intraovarian factors and steroid hormones. Bone morphogenic protein 4 (BMP4) as a follicle survival factor can promote primordial follicle development (Nilsson and Skinner, 2003). Folliculogenesis is tightly associated with follicle apoptosis. Uncontrolled follicle apoptosis would reduce the number of available follicles (Sun et al., 2012). The ratio of pro-apoptotic factor bcl-2 associated $\mathrm{X}$ protein (BAX) and anti-apoptotic factor B cell lymphoma/leukemia2 (Bcl-2) serves as a good marker for ovarian follicle apoptosis (Rucker et al., 2000). Proliferating cell nuclear antigen (PCNA) regulates primordial follicle assembly in neonatal mouse ovaries by promoting apoptosis of oocytes (Xu et al., 2011). In addition, sex steroid hormones, such as estrogen and progesterone (P4), can inhibit early follicle development in rodents (Kezele and Skinner, 2003; Chen et al., 2007). Steroidogenesis, the biosynthesis of steroid hormones, is determined by a number of steroidogenic factors and enzymes. Steroidogenesis in ovary is regulated by pituitary gonadotropic hormones follicle-stimulating hormone (FSH) and luteinizing hormone (LH) through their respective receptors located on ovarian follicles (Camp et al., 1991) and can also be modulated by BMP15 which is produced by the oocytes (Sun et al., 2010). It is suggested that folliculogenesis and steroidogenesis in neonatal ovary are closely related processes which are sensitive to prenatal environment (Grzesiak et al., 2012). However, knowledge is lacking about how maternal nutrition affects the expression of folliculogenic and steroidogenic genes in the ovary of neonatal animals.

Maternal LP diet throughout gestation can induce gene promoter methylation which regulates gene expression in the transcriptional level (Jia et al., 2012). MicroRNAs (miRNAs) play important roles in post-transcriptional regulation of gene expression through decreasing mRNA stability or inhibiting translation (Fire et al., 1998). The regulatory roles of miRNAs in fetal ovarian development (Torley et al., 2011), steroidogenesis (Sirotkin et al., 2009) and granulosa cells apoptosis (Fiedler et al., 2008) have been explored recently (Baley and Li, 2012). However, it remains unclear whether miRNAs are involved in the effect of maternal malnutrition on the expression of folliculogenic and steroidogenic genes in the ovary of neonatal animals.

Therefore, the present study was designed to explore the effects of maternal LP diet on the expression of genes involved in folliculogenesis and steroidogenesis in neonatal pig ovary, and to evaluate whether miRNAs are involved in these processes. The results will be helpful in increasing our knowledge on the mechanism underlying the impact of maternal nutrition on ovarian development in neonatal animals.

\section{MATERIALS AND METHODS}

\section{Animals}

The Animal Ethics Committee at Nanjing Agricultural University reviewed the protocol and approved this study specifically, with the project number 2012ZX08009-103B. The slaughter and sampling procedures complied with the "Guidelines on Ethical Treatment of Experimental Animals" (2006) No. 398 set by the Ministry of Science and Technology, China.

The animal treatments and sampling methods were according to a previously published method (Jia et al., 2012). LandracexYorkshire crossbred, second parity sows were artificially inseminated, at the observation of estrus, with a mixture of Duroc semen samples obtained from two littermate boars. One week after artificial insemination, sows were randomly divided into standard-protein (SP) and LP diet groups. The SP sows were fed diets containing $15 \%$ of crude protein throughout gestation and those in the LP group were fed diets containing $7.5 \%$ of crude protein. The diet composition is shown in Table 1. All sows were kept in the same room with a constant temperature maintained at $25^{\circ} \mathrm{C}$ and a $12-\mathrm{h}$ light/dark cycle. Sows were fed three times

Table 1. Ingredients and calculated composition of the experimental diets

\begin{tabular}{lcc}
\hline Items & Standard protein & Low-protein \\
\hline Ingredient (g/kg) & 370 & 615 \\
Corn & 300 & 100 \\
Wheat & 80 & 100 \\
Bran & 170 & 0 \\
Soybean Meal & 0 & 100 \\
Maize starch & 30 & 40 \\
Lignocellulose & 20 & 25 \\
CaHPO4 & 10 & 0 \\
Soybean oil & 20 & 20 \\
Premix* & & \\
Calculated composition & 13.10 & 13.10 \\
Digestible energy (MJ/kg) & 15 & 7.50 \\
Crude protein $(\%)$ & 4.50 & 4.30 \\
Crude fiber $(\%)$ & 0.84 & 0.85 \\
Calcium (\%) & 0.65 & 0.61 \\
Phosphorous $(\%)$ &
\end{tabular}

* The premix contains (per kilogram): retinol: 1,100 kIU; cholecalciferol, $350 \mathrm{kIU}$; vitamin $\mathrm{K}_{3}, 0.4 \mathrm{~g}$; vitamin $\mathrm{B}_{1}, 0.4 \mathrm{~g}$; vitamin $\mathrm{B}_{2}, 1,640 \mathrm{mg}$; vitamin $\mathrm{B}_{6}, 0.65 \mathrm{~g}$; vitamin $\mathrm{B}_{12}, 4.4 \mathrm{mg}$; lysine, $72 \mathrm{~g}$; niacin, $4.5 \mathrm{~g}$; pantothenic acid, $2.5 \mathrm{~g}$; d-pantothenic acid, $2 \mathrm{~g}$; folic acid, $5.2 \mathrm{~g}$; biotin, $30 \mathrm{mg}$; d-biotin, $16 \mathrm{mg}$; choline chloride, $30 \mathrm{~g}$; vitamin C, $20 \mathrm{~g}$; manganese, $0.8 \mathrm{~g}$; zinc, $7 \mathrm{~g}$; ferrous, $7 \mathrm{~g}$; copper, $2 \mathrm{~g}$; selenium, $20 \mathrm{mg}$; sodium chloride, $3 \mathrm{~g}$; $\beta$-xylanase, 8,000 kIU; antioxidant, $0.19 \mathrm{~g}$; acidifier, $2.5 \mathrm{~g}$. 
daily (05:00, 10:00, and 17:00 h) and had free access to water. The farrowing duration and litter size of sows, sex ratio and death ratio of newborn piglts were not affected by the maternal diet. Eight sows $(\mathrm{n}=8)$ in each group were chosen in the experiment. Newborn piglets were individually weighed immediately after parturition.

\section{Tissue collection}

The piglets of the same litter were gathered and confined in the warm creep area. One female piglet of the mean body weight $( \pm 10 \%)$ was selected from each litter and exsanguinated before suckling. Blood was collected immediately and serum was separated by centrifugation at $3,000 \times g$ for $10 \mathrm{~min}$ at $4^{\circ} \mathrm{C}$ and stored at $-20^{\circ} \mathrm{C}$. Both ovaries were collected within $20 \mathrm{~min}$, snap-frozen in liquid nitrogen and stored at $-80^{\circ} \mathrm{C}$ for subsequent RNA and DNA extraction.

\section{Hormone assays}

The serum concentrations of $\mathrm{P} 4$, testosterone $(\mathrm{T})$ and $17 \beta$-estradiol (E2) were measured using respective commercial ${ }^{125}$ I-RIA kits (Catalog no. B08PZB, B10PZB and $\mathrm{B} 05 \mathrm{PZB}$, respectively, Beijing North Institute of Biological Technology, Beijing, China) according to the manufacturer's manuals. The limit of detection was 0.05 $\mathrm{ng} / \mathrm{mL}$ for $\mathrm{P} 4,0.02 \mathrm{ng} / \mathrm{mL}$ for $\mathrm{T}$, and $5 \mathrm{pg} / \mathrm{mL}$ for E2. The intra-assay coefficient was $10 \%$ for all the assays. The cross reactivity of $\mathrm{T}$ Radioimmunoassay (RIA) was $0.011 \%$ with dihydrotestosterone, $0.021 \%$ with $\mathrm{E} 2,0.032 \%$ with $\mathrm{P} 4$, and less than $0.01 \%$ with androstenedione and estriol. The cross reactivity of E2 RIA was $0.016 \%$ with estriol, $0.01 \%$ with T and less than $0.01 \%$ with $\mathrm{P} 4$. All samples were measured in one assay in duplicate.

\section{RNA extraction and reverse transcription}

Total RNA was extracted from homogenized ovaries using TRIzol Reagent (Invitrogen, Grand Island, New York, USA) and then treated with DNase I (RNase Free, D2215, Takara, Dalian, China) to eliminate possible contamination of genomic DNA according to the manufacturer's instructions. Concentration of the extracted RNA was measured using NanoDrop 1000 Spectrophotometer (ND1000; Thermo, Wilmington, DE, USA). Ratios of absorption $(260 / 280 \mathrm{~nm})$ were between 1.9 and 2.1. RNA integrity was confirmed by denaturing agarose electrophoresis, and DNA contamination was examined by polymerase chain reaction (PCR). Two micrograms of total RNA were reverse-transcribed in a final volume of $25 \mu \mathrm{L}$ with M-MLV reverse transcriptase (Promega, Madison, WI, USA) and random hexamer primers (Takara, China) following the manufacturer's instructions. Reverse transcription was performed in a Thermal Cycler PTC0200
(Bio-Rad, Hercules, CA, USA).

\section{Real-time polymerase chain reaction}

Two microliters of diluted cDNA (1:20) were used for real-time PCR to quantitate the expression of folliculogenic genes including $B A X, B c l-2, B M P 15, B M P 4$, and PCNA, steroidogenic genes including steroidogenic acute regulatory protein (StAR), cytochrome $\mathrm{P} 450$ cholesterol side-chain cleavage enzyme (CYP11A1), 3 $\beta$-hydroxysteroid dehydrogenase (3ßHSD), cytochrome P450 17ahydroxylase (CYP17A1) and cytochrome P450 aromatase (CYP19A1), genes encoding receptors including folliclestimulating hormone receptor (FSHR), luteinizing hormone receptor (LHR), estrogen receptor $\alpha(\mathrm{ER} \alpha)$ and ER $\beta$. All primers (Table 2) were designed with Primer 5 software according to their mRNA sequences and synthesized by Generay Biotech Co., Ltd. (Shanghai, China). Real-time PCR was performed by Mx3000P (Stratagene, Santa Clara, CA, USA). 18S rRNA was chosen as reference gene. The amplification specificity of each gene was checked by melting curve analysis. The PCR products were validated by sequencing.

\section{Quantitation of miRNAs targeting CYP19A1, FSHR, BMP4 and PCNA}

The 3' untranslated regions (3'UTR) or the 3' flanking sequence of relevant genes was acquired from ensembl database (http://www.ensembl.org/). All the porcine miRNAs gene sequences were acquired from miRBase (http://www.mirbase.org/). Because 3' UTR sequences of porcine CYP19A1 and FSHR genes have not been reported, we aligned the 3 ' flanking sequences of these two genes with the respective human 3 ' UTR sequences to get the consensus sequences for further prediction. The PITA (http://genie.weizmann.ac.il/pubs/mir07/mir07_prediction.h tml) algorithm (Kertesz et al., 2007) was applied to predict the miRNAs targeting BMP4, PCNA, CYP19A1, and FSHR with the threshold of score set at -10 .

Total RNA was treated with DNase I (RNase Free, D2215, Takara, Japan), and six micrograms of treated total RNA were polyadenylated by poly (A) polymerase at $37^{\circ} \mathrm{C}$ for $1 \mathrm{~h}$ in a $25 \mu \mathrm{L}$ reaction mixture using Poly (A) Tailing Kit (AM1350, Ambion, Grand Island, New York, USA) according to the manufacturer's instructions. Two micrograms of polyadenylated RNA were reverse transcribed using poly (T) adapter. Real-time PCR was performed with a miRNA-specific forward primer identical to the miRNA sequence except that the uracil being replaced by thymine, and a universal reverse primer complementary to part of the poly (T) adapter sequence. small nuclear RNA (U6 snRNA) was used as a reference gene to normalize the expression of miRNAs. The 
Table 2. Nucleotide sequences of specific primers

\begin{tabular}{|c|c|c|c|}
\hline Gene name & Products (bp) & Primer sequences (5' to 3') & GenBank accession number \\
\hline $18 S$ rRNA & 122 & $\begin{array}{l}\text { F: CCCACGGAATCGAGAAAGAG } \\
\text { R: TTGACGGAAGGGCACCA }\end{array}$ & AY265350.1 \\
\hline$B A X$ & 143 & $\begin{array}{l}\text { F: TGACGGCAACTTCAACTGGG } \\
\text { R: GCAGCCGATCTCGAAGGAA }\end{array}$ & XM_003127290.2 \\
\hline$B c l-2$ & 142 & $\begin{array}{l}\text { F: GCCTTTGTGGAGCTGTATGG } \\
\text { R: CCCGTGGACTTCACTTATGG }\end{array}$ & XM_003121700.2 \\
\hline BMP15 & 235 & $\begin{array}{l}\text { F: TCAGAGCCACTGTGGTTTATCG } \\
\text { R: CACATGAAGCGGAGTCGTAGAA }\end{array}$ & NM_001005155.1 \\
\hline$B M P 4$ & 172 & $\begin{array}{l}\text { F: GGGCTCGGAAGAAGAATAAGA } \\
\text { R: ACGATGGCATGATTGGTTGA }\end{array}$ & NM_001101031.2 \\
\hline PCNA & 90 & $\begin{array}{l}\text { F: GGTTCAGGCTCTGTTGTGGTGT } \\
\text { R: GCTACAGGTGGAAGAAAGAAGGAA }\end{array}$ & XM_003359883.1 \\
\hline $3 \beta H S D 1$ & 145 & $\begin{array}{l}\text { F: CACTGACCTGGGCTGATGAC } \\
\text { R: GTGGCGAGAAGCAGACAAGA }\end{array}$ & NM_001004049.1 \\
\hline $17 \beta H S D 2$ & 196 & $\begin{array}{l}\text { F: TCGAGCAGACGTTCCTTGAG } \\
\text { R: TGGTCGCCGAACACTCTTT }\end{array}$ & NM_001128472.1 \\
\hline CYP19A1 & 187 & $\begin{array}{l}\text { F: GCTGCTCATTGGCTTAC } \\
\text { R: TCCACCTATCCAGACCC }\end{array}$ & NM_214431.1 \\
\hline CYP17A1 & 136 & $\begin{array}{l}\text { F: TCCAAGCCAAGACGAAC } \\
\text { R: TTTACCACAGAGGCAGAAG }\end{array}$ & NM_214428.1 \\
\hline CYP11A1 & 149 & $\begin{array}{l}\text { F: GGCTCCAGAGGCCATAAAGA } \\
\text { R: ACTCAAAGGCGAAGCGAAAC }\end{array}$ & $\mathrm{X} 13768.1$ \\
\hline StAR & 202 & $\begin{array}{l}\text { F: GACTTTGTGAGTGTCGGCTGTA } \\
\text { R: ATCCCTTGAGGTCAATGCTG }\end{array}$ & U53020.1 \\
\hline FSHR & 152 & $\begin{array}{l}\text { F: TCACAGTCCCTCGGTTCCTT } \\
\text { R: AGCATCACAGCCTGCTCCA }\end{array}$ & NM_214386.2 \\
\hline$L H R$ & 255 & $\begin{array}{l}\text { F: ATGGGGCTCTACCTGCTACTCA } \\
\text { R: GAGCCACCСTCCAAGCATAA }\end{array}$ & NM_214449.1 \\
\hline$E R \alpha$ & 151 & $\begin{array}{l}\text { F: ATGAAGTGCAAGAACGTGGTG } \\
\text { R: AATGCGATGGAGTTGAGCC }\end{array}$ & NM_214220.1 \\
\hline$E R \beta$ & 191 & $\begin{array}{l}\text { F: GCCCTGTTACCAGTCCAAGTT } \\
\text { R: ACGCCGGTTCTTATCTATCGT }\end{array}$ & NM_001001533.1 \\
\hline
\end{tabular}

F, forward; R, reverse; BAX, bcl-2 associated X protein; Bcl-2, B cell lymphoma/leukemia-2; BMP, bone morphogenic protein; PCNA, proliferating cell nuclear antigen; $\beta \mathrm{HSD}, \beta$-hydroxysteroid dehydrogenase; CYP19A1, cytochrome P450 aromatase; CYP17A1, cytochrome P450 17a-hydroxylase; CYP11A1, cytochrome P450 cholesterol side-chain cleavage enzyme; StAR, steroidogenic acute regulatory protein; FSHR, follicle-stimulating hormone receptor; LHR, luteinizing hormone receptor; ER, estrogen receptor $\alpha$.

sequences of all the primers and poly ( $\mathrm{T}$ ) adapter were listed in Table 3.

\section{DNA extraction and methylated DNA} immunoprecipitation analysis

DNA was extracted using TRIzol reagent together with RNA extraction process according to the manufacturer's instruction. Methylated DNA immunoprecipitation (MeDIP) analysis was performed as previously described (Liu et al., 2011; Jia et al., 2012). The isolated DNA from ovary was sonicated to produce small fragments (300 to 500 bp). Two micrograms of fragmented DNA was denatured for $10 \mathrm{~min}$ in boiling water and diluted to the final concentration of $20 \mathrm{ng} / \mu \mathrm{L}$. The volume of $100 \mu \mathrm{L}$ of the denatured DNA was stored as input. A mouse monoclonal antibody against 5-methyl cytidine (No. ab10805, Abcam) was used to immunoprecipitate methylated DNA fragments. The immune complexes were captured with protein $G$ agarose beads $(40 \mu \mathrm{L}, 50 \%$ slurry, pretreated with denatured salmon sperm DNA). The beads bound to immune complexes were washed to eliminate nonspecific binding and resuspended in $250 \mu \mathrm{L}$ digestion buffer containing proteinase K. Finally, the MeDIP DNA was purified. The methylation control 1 (mc1) was used as an internal reference and its primers were designed to amplify a sequence of $\beta$-actin promoter which is absent of cytosine phosphate guanine $(\mathrm{CpG})$ sites. The volume of $2 \mu \mathrm{L}$ of MeDIP DNA and input DNA respectively was used to amplify the proximal promoter sequences of relevant genes by real-time PCR with specific primers designed with Primer 5 software (Table 4). 
Table 3. The primer sequences of miRNAs expression

\begin{tabular}{lll}
\hline Name & \multicolumn{1}{c}{ Primer sequences (5' to 3') } & miRbase accession \\
\hline miR-378 & ACTGGACTTGGAGTCAGAAGGC & MIMAT0013868 \\
miR-98 & TGAGGTAGTAAGTTGTATTGTT & MIMAT0013905 \\
miR-140-5p & AGAGGTAGTAGGTTGCATAGTT & MIMAT0025356 \\
miR-140-3p & AGTGGTTTTACCCTATGGTAG & MIMAT0002143 \\
miR-let-7c & TACCACAGGGTAGAACCACGGAC & MIMAT0013881 \\
miR-423-5p & TGAGGTAGTAGGTTGTATGGTT & MIMAT0002151 \\
miR-423-3p & TGAGGGGCAGAGAGCGAGACTTT & MIMAT0013880 \\
miR-17-5p & AGCTCGGTCTGAGGCCCCTCAGT & MIMAT0013881 \\
miR-17-3p & CAAAGTGCTTACAGTGCAGGTAG & MIMAT0007755 \\
miR-885-3p & ACTGCAGTGAAGGCACTTGTAG & MIMAT0015268 \\
miR-421-5p & AGGCAGCGGGGTGTAGTGGAT & MIMAT0013903 \\
miR-18a & CCTCATTAAATGTTTGTTGAATGA & MIMAT0017970 \\
miR-122 & TAAGGTGCATCTAGTGCAGATA & MIMAT0002161 \\
miR-210 & TGGAGTGTGACAATGGTGTTTGT & MIMAT0002119 \\
miR-487b & CTGTGCGTGTGACAGCGGCTGA & MIMAT0007761 \\
U6 snRNA & GTGGTTATCCCTGTCCTGTTCG & MIMAT0017973 \\
poly(T) adapter & GGCAAGGATGACACGCAAAT & ENSSSCT00000019750 \\
& TAGAGTGAGTGTAGCGAGCACAGA & \\
universal primer & ATTAATACGACTCACTATAGGTTTTT & \\
\hline
\end{tabular}

\section{Statistical analysis}

All data are presented as mean \pm standard error of the mean and were analyzed using independent samples t-test with SPSS 17.0 for windows. The method of $2^{-\Delta \Delta \mathrm{Ct}}$ was used to analyze the real-time PCR data, and the mRNA levels were expressed as the fold change relative to the mean value of the SP group. The differences were considered statistically significant when $p<0.05$.

\section{RESULTS}

\section{Ovary weight and serum steroid hormones}

As shown in Table 5, maternal dietary protein restriction did not affect the birth weight of offspring piglets, yet the

Table 4. The primer sequences of MeDIP analysis

\begin{tabular}{ll}
\hline Name & \multicolumn{1}{c}{ Primer sequences (5' to 3') } \\
\hline BMP4 CpG1 & F: AACTGGGAACAGGCTTAGGAA \\
& R: GACCCTCGAAACTGAATCTGC \\
BMP4 CpG2 & F: GACCTGCCTTCCAGAGTCCA \\
& R: CCGCAGTCAAGTGGGAGAA \\
PCNA CpG & F: CCGCATCTGCAACCTATACCA \\
& R: CGAATCCGACTAGGAACCATG \\
CYP19A1 CpG & F: ATCGTGGAGCAGTGGTTA \\
& R: GTCGAATCGGAGCCTTAG \\
mc1 & F: CCCTATAACGCCTTGCCAAACC \\
& R: GGGTAGGTGCCTGCTTTCGTAG \\
\hline
\end{tabular}

MeDIP, methylated DNA immunoprecipitation; F, forward; R, reverse; BMP, bone morphogenic protein; PCNA, proliferating cell nuclear antigen; CYP19A1, cytochrome P450 aromatase; mc1, methylation control 1. ovary weight tended to be lower $(\mathrm{p}=0.07)$ and the ovary weight relative to body weight was significantly decreased $(\mathrm{p}<0.05)$ in the LP group. No significant differences were detected for serum $\mathrm{P} 4$ or $\mathrm{T}$ concentrations between the two groups, but serum E2 level was significantly higher $(p<0.05)$ in LP offspring. These results indicate disrupted ovarian development in neonatal offspring born to undernourished sows.

\section{Ovary expression of folliculogenic and steroidogenic genes}

The LP piglets expressed significantly higher BAX $(\mathrm{p}<0.05)$ but lower Bcl-2 $(\mathrm{p}<0.05)$ mRNA in the ovary, which resulting in higher ratio of $\mathrm{BAX} / \mathrm{Bcl}-2(\mathrm{p}<0.01)$. Moreover, mRNA expression of BMP4 $(\mathrm{p}<0.05)$ and PCNA

Table 5. Body weight, ovary weight and serum concentrations of sex steroid hormones in neonatal piglets

\begin{tabular}{lccc}
\hline Parameter & SP & LP & p value \\
& $\mathrm{n}=8$ & $\mathrm{n}=8$ & \\
\hline $\mathrm{BW}(\mathrm{kg})$ & $1.49 \pm 0.08$ & $1.57 \pm 0.04$ & 0.40 \\
OW $(\mathrm{g})$ & $0.11 \pm 0.01$ & $0.08 \pm 0.01$ & 0.07 \\
OW/BW $(\mathrm{g} / \mathrm{kg})$ & $0.08 \pm 0.01$ & $0.05 \pm 0.01$ & 0.03 \\
$\mathrm{E} 2(\mathrm{pg} / \mathrm{mL})$ & $6.42 \pm 2.12$ & $38.33 \pm 17.57$ & 0.04 \\
$\mathrm{P} 4(\mathrm{ng} / \mathrm{mL})$ & $9.10 \pm 1.42$ & $13.56 \pm 2.94$ & 0.16 \\
$\mathrm{~T}(\mathrm{ng} / \mathrm{mL})$ & $0.42 \pm 0.38$ & $1.16 \pm 0.68$ & 0.33 \\
\hline
\end{tabular}

SP, standard protein; LP, low-protein; BW, body weight; OW, ovary weight; E2, 17ß-estradiol; P4, progesterone; T, testosterone; SEM, standard error of the mean.

Values are mean \pm SEM, statistical significances were set at $\mathrm{p}<0.05$. 
$(\mathrm{p}<0.05)$ were significantly up-regulated in LP group (Figure 1A). Among seven steroidogenic genes detected, CYP17A1 ( $p=0.05)$ tended to be lower, and CYP19A1 $(p<0.05)$ were significantly down-regulated in LP piglets. Ovarian expression of ER $\alpha / \beta, 3 \beta \mathrm{HSD}, 17 \beta \mathrm{HSD} 1$, CYP11A1 and StAR mRNA in neonatal piglets was not affected by maternal dietary protein restriction. The alterations in ovarian expression of folliculogenic and steroidogenic genes were associated with significantly down-regulated FSHR mRNA expression $(\mathrm{p}<0.05)$ in the ovary of LP piglets (Figure 1B). These results suggest that maternal protein restriction affects the expression of folliculogenic and steroidogenic genes, which are related to follicle apoptosis and survival, in the ovary of neonatal offspring piglets.

Methylated DNA immunoprecipitation analysis in offspring neonatal piglets

To determine whether the alterations in ovarian gene expression involve modifications in $\mathrm{CpG}$ methylation on respective gene promoters, we employed MeDIP assay using specific primers to amplify the fragments spanning most of the $\mathrm{CpG}$ islands on gene promoters (Figure 2). However, no differences were detected in the methylation levels on BMP4, PCNA, or CYP19A1 gene promoters between SP and LP neonatal piglets ovary.

\section{Expression of miRNAs predicted to target CYP19A1, FSHR, BMP4, and PCNA}

Nine miRNAs for CYP19A1, three for BMP4, two for PCNA and three for FSHR were chosen for miRNA quantification. We found that the expression of miR-423-5p targeting CYP19A1 and PCNA, miR-378 targeting CYP19A1 and miR-210 targeting BMP4 were significantly down-regulated $(\mathrm{p}<0.05)$ in the ovary of LP offspring. Moreover, the expression of miR-423-3ptargeting FSHR tended to be lower $(\mathrm{p}=0.08)$ in the ovary of LP piglets (Figure 3). These results implicate that miRNAs-mediated post-transcriptional gene regulation is involved in the effects of maternal protein restriction on ovarian expression of folliculogenic and steroidogenic genes in the neonatal piglets.

\section{DISCUSSION}

To our knowledge, this is the first report on the effect of maternal LP diet on ovarian genes, miRNAs expression and MeDIP analysis in neonatal piglets. Alterations in the ovarian expression of folliculogenic and steroidogenic genes suggest the potential impact of maternal LP diet on

A

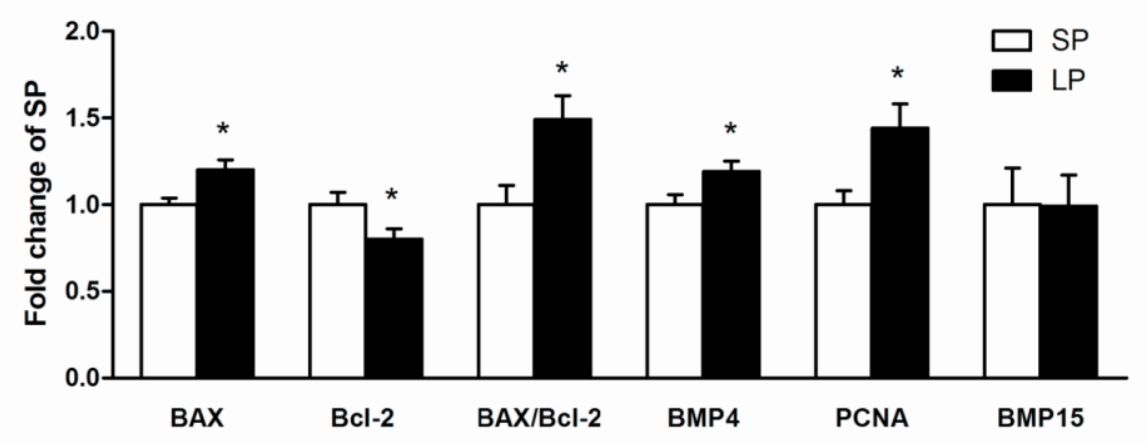

B

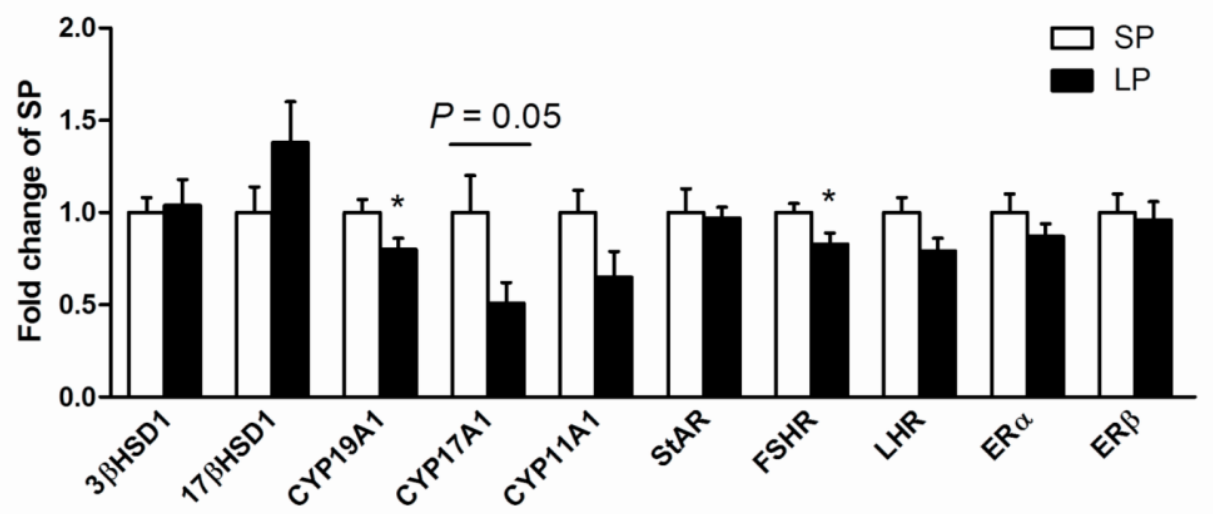

Figure 1. Ovarian expression of folliculogenic and steroidogenic genes in neonatal piglets. A, folliculogenic genes expression; B, steroidogenic genes expression. Data are expressed as mean \pm standard error of the mean. Asterisks indicate statistically significant differences $(\mathrm{p}<0.05), \mathrm{n}=8$. 
A
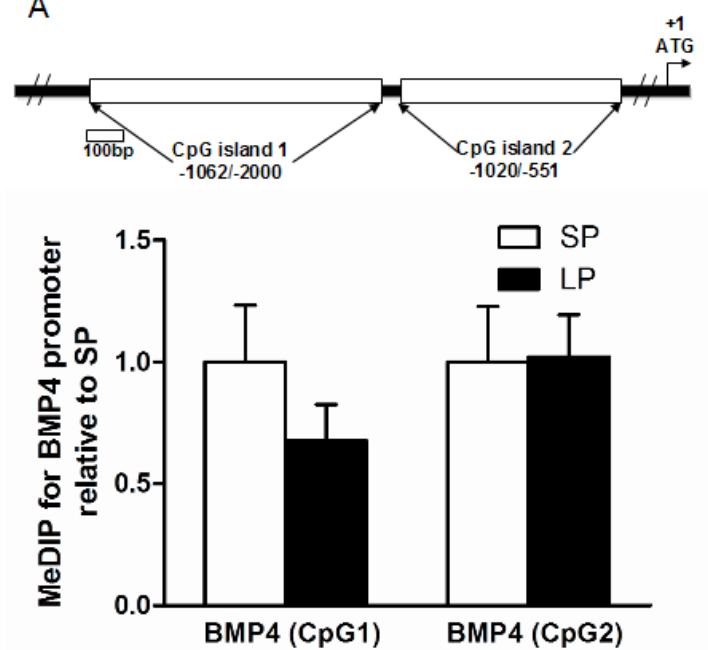

B
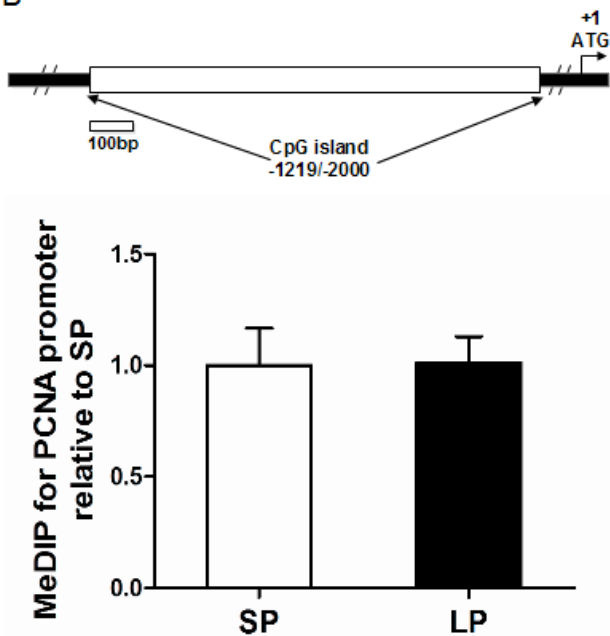

C
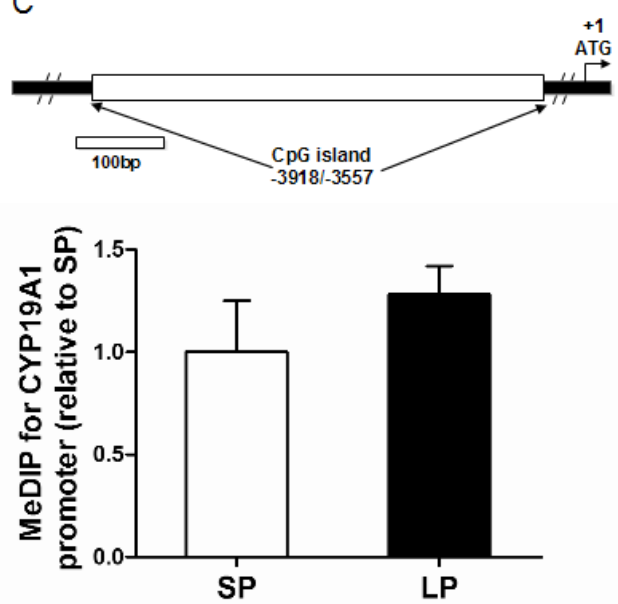

Figure 2. DNA methylation analysis in the $\mathrm{CpG}$ islands of BMP4, PCNA, and CYP19A1 promoter. The CpG islands in the promoter of each gene are presented above the bargraphs of respective MeDIP analysis. A, CpG islands of BMP4 promoter and MeDIP analysis; B, CpG island of PCNA promoter and MeDIP analysis; C, CpG island of CYP19A1 promoter and MeDIP analysis. Data are expressed as mean \pm standard error of the mean. Asterisks indicate statistically significant differences $(\mathrm{p}<0.05), \mathrm{n}=8$. CpG, cytosine phosphate guanine; BMP4, bone morphogenic protein 4; PCNA, proliferating cell nuclear antigen; CYP19A1, cytochrome P450 aromatase; MeDIP, methylated DNA immunoprecipitation.

ovarian development and function in neonatal piglets. Moreover, we provide preliminary evidences that miRNAs predicted to target folliculogenic and steroidogenic genes are involved in mediating the effect of maternal LP diet on ovarian function in newborn piglets.

Numerous studies in rats indicate that maternal malnutrition during pregnancy decreases ovarian weight in adults (da Silva Faria et al., 2004; Iwasa et al., 2011). In this study, disrupted ovarian development in prenatally undernourished offspring was observed in neonatal age; LP piglets demonstrated significantly lower ovary weight relative to body weight at birth. Sex steroid hormones are crucial for the development of ovary. In the present study, we examined the levels of sex steroid hormones and found that serum E2 concentration was higher in LP group. Other studies also reported significantly increased serum E2 concentration in 40-day-old rats subjected to maternal protein restriction during lactation (da Silva Faria et al., 2008; Faria et al., 2010). Primary follicles are the main sites for estrogen production in the neonatal animals, so higher E2 concentration may implicate increased number of primary follicles in the ovary of neonatal LP piglets (Faria et al., 2010).

The PCNA, a proliferation factor, is recently found to play an important role in ovarian folliculogenesis (Xu et al., 2011). In pigs, PCNA is expressed in growing follicles, but not in quiescent primordial follicles (Tománek and Chronowska, 2006). In the ovary of neonatal piglets, follicle development is mainly the transition from primordial to primary follicle (Ding et al., 2010). In this 
A
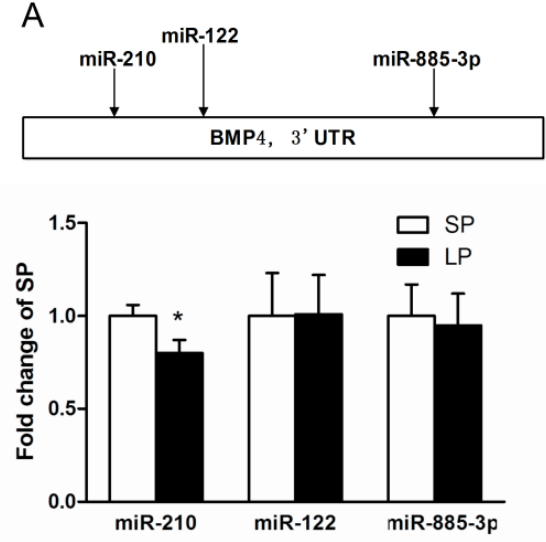

C
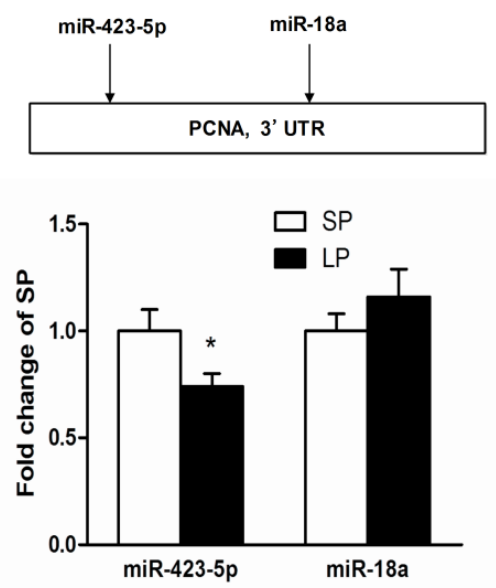

B
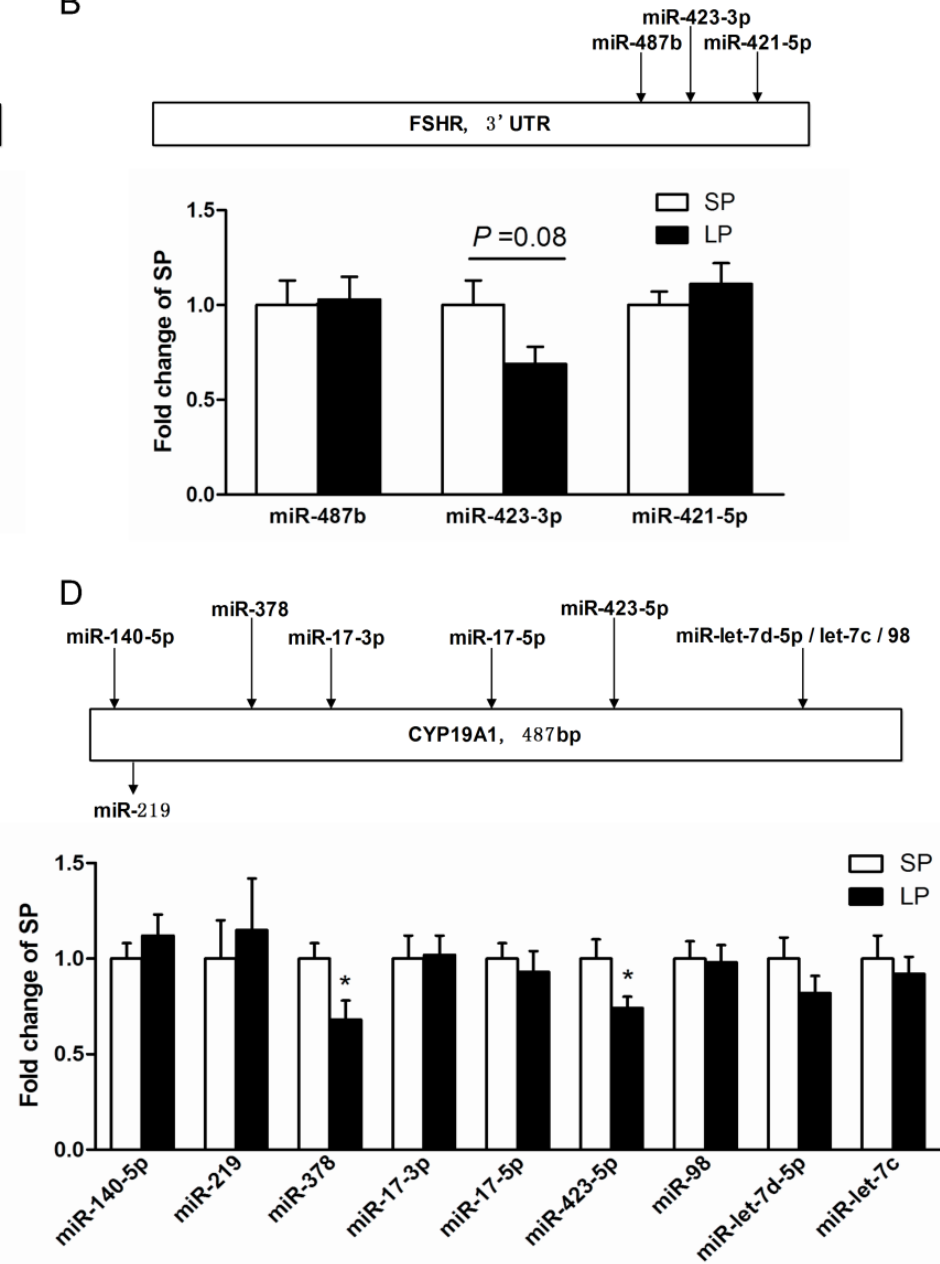

Figure 3. Ovarian expression of miRNAs predicted to target A, BMP4; B, FSHR; C, PCNA; and D, CYP19A1 genes in neonatal piglets. Predicted binding sites of miRNAs on each target gene are presented above the bargraphs of respective miRNA expression. Data are expressed as mean \pm standard error of the mean. Asterisks indicate statistically significant differences $(\mathrm{p}<0.05), \mathrm{n}=8$. BMP4, bone morphogenic protein 4; FSHR, follicle-stimulating hormone receptor; PCNA, proliferating cell nuclear antigen; CYP19A1, cytochrome P450 aromatase.

study, PCNA mRNA abundance was significantly higher in LP group than the SP group, suggesting that the ovary in neonatal LP piglets contains more primary follicles. This finding agrees with early reports that knock-down of PCNA expression by RNA interference retarded the transition of primordial follicles into primary follicles in vitro (Xu et al., 2011). The BMP4 can also promote the transition of primordial-to-primary follicles. Neonatal rat ovaries treated with exogenous BMP4 had a higher proportion of primary follicles and fewer primordial follicles (Nilsson and Skinner, 2003). Conversely, immune-neutralization with BMP-4 antibody led to lower primary follicles and higher primordial follicles in mouse ovaries (Tanwar et al., 2008). Here, we found that the change of BMP4 expression was consistent with PCNA. These findings indicate that the ovary development in neonatal LP piglets may be accelerated. It also may help to explain the observations that maternal undernutrition in pregnancy results in premature aging of offspring ovary (Bernal et al., 2010) and an earlier termination of breeding capacity (da Silva Faria et al., 2008).

Apoptosis is a physiological process which is required for primordial follicle assembly and early follicle development (Ding et al., 2010). The PCNA can also regulate primordial follicle assembly in neonatal mouse ovaries by promoting apoptosis of oocytes (Xu et al., 2011). In mammals, follicle apoptosis or follicular atresia begins from early fetal period with most follicles lost at birth (Ding et al., 2010). These reports suggest that excessive ovarian follicular development in neonatal piglets accompanied with increased apoptosis of follicle. The ratio of proapoptotic factor BAX and antiapoptotic factor Bcl-2 is an evaluation index for the level of apoptosis (Rucker et al., 2000; Sun et al., 2012). In this study, the ratio of BAX/Bcl- 
2 was higher in the ovary of LP piglets. The enhanced follicle apoptosis further supporting our presumption that maternal LP diet advances the early follicle development in the ovary of neonatal pigs.

Estrogen has multiple effects on the development of ovarian follicles through its receptors $(E R \alpha / \beta)$. Studies on rat ovaries indicate that E2 inhibits primordial to primary follicle transition both in vivo and in vitro (Kezele and Skinner, 2003). In this study, ovarian expression of $E R \alpha / \beta$ mRNA was not altered, despite significantly elevated serum E2 concentration in LP piglets. The elevated serum E2 level may result from accelerated ovary development in LP piglets. Therefore, enhanced steroidogenesis may be expected in the ovary of LP piglets. On the contrary, the ovarian mRNA expression of estrogen biosynthesis genes, such as CYP17Al and CYP19A1, tended to be or are significantly down-regulated in LP piglets, which was associated with a significant down-regulation of FSHR mRNA. This contradicts with the elevated serum E2 and advanced ovarian follicular development in LP piglets. In addition, there is no difference in the DNA methylation of PCNA, BMP4, and CYP19A1 promoter. So it is possible that the protein content or the enzyme activity is uncoupled with the mRNA abundance, with the possible involvement of post-transcriptional regulation. Unfortunately, we were not able to detect the protein content or the enzyme activity because neonatal ovaries are not big enough for both RNA and protein extractions.

However, we detected the miRNA regulation, and found that the expression of miR-423-5p and miR-378 targeting CYP19A1 was significantly downregulated, implying that the post-transcriptional repression of CYP19A1 may be decreased which thereby results in higher CYP19A1 protein content or enzyme activity. Similar alterations are also detected for miRNAs targeting folliculogenic genes. MiR423-5p and miR-210 which are predicted to target PCNA and BMP4 respectively were also found to be significantly down-regulated in the ovary of LP piglets, suggesting an inhibitory role of miRNAs in mRNA stability. It is noted that miR-423-5p is predicted to target both CYP19A1 and PCNA, but the regulatory mechanisms may be different, being translation inhibition for CYP19A1 while mRNA degradation for PCNA. We did not detect alterations in the expression of the two miRNAs predicted to target porcine FSHR gene, but whether other miRNAs are involved in the regulation of FSHR remains elusive. There is no 3' UTR sequence reported for porcine CYP17Al, BAX, and Bcl-2 genes, therefore we are not able to predict their regulatory miRNAs from 3' UTR sequences.

In conclusion, our results suggest that a maternal LP diet in pregnancy affects the expression of folliculogenic and steroidogenic genes in neonatal piglets. Posttranscriptional regulation of gene expression by miRNAs plays an important role in these processes. Neonatal changes in ovarian function may persist to adulthood, with long-term consequences on folliculogensis and steroidogensis. Our findings may shed light on understanding the ovarian aging and ovarian reserve reduction in prenatally undernourished offspring.

\section{ACKNOWLEDGMENTS}

This work was supported by the National Basic Research Program of China (2012CB124703, 2014CB138502), the Special Fund for Agro-scientific Research in the Public Interest (201003011), the Fundamental Research Funds for the Central Universities (KYZ200913) and the Priority Academic Program Development of Jiangsu Higher Education Institutions.

\section{REFERENCES}

Baley, J. and J. Li. 2012. Micrornas and ovarian function. J. Ovarian Res. 5:8.

Bernal, A. B., M. H. Vickers, M. B. Hampton, R. A. Poynton, and D. M. Sloboda. 2010. Maternal undernutrition significantly impacts ovarian follicle number and increases ovarian oxidative stress in adult rat offspring. Plos One. 5(12):e15558.

Camp, T. A., J. O. Rahal, and K. E. Mayo. 1991. Cellular localization and hormonal regulation of follicle-stimulating hormone and luteinizing hormone receptor messenger rnas in the rat ovary. Mol. Endocrinol. 5:1405-1417.

Chen, Y., W. N. Jefferson, R. R. Newbold, E. Padilla-Banks, and M. E. Pepling. 2007. Estradiol, progesterone, and genistein inhibit oocyte nest breakdown and primordial follicle assembly in the neonatal mouse ovary in vitro and in vivo. Endocrinology 148:3580-3590.

da Silva Faria, T., C. da Fonte Ramos, and F. J. B. Sampaio. 2004. Puberty onset in the female offspring of rats submitted to protein or energy restricted diet during lactation. J. Nutr. Biochem. 15:123-127.

da Silva Faria, T., F. de Bittencourt Brasil, F. J. Sampaio, and C. da Fonte Ramos. 2008. Maternal malnutrition during lactation alters the folliculogenesis and gonadotropins and estrogen isoforms ovarian receptors in the offspring at puberty. J. Endocrinol. 198:625-634.

Ding W., W. Wang, B. Zhou, W. Zhang, P. Huang, F. Shi, and K. Taya. 2010. Formation of primordial follicles and immunolocalization of PTEN, PKB and FOXO3a proteins in the ovaries of fetal and neonatal pigs. J. Reprod. Dev. 56:162168.

Durlej, M., K. Knapczyk-Stwora, M. Duda, J. Galas, and M. Slomczynska. 2011. The expression of FSH receptor (FSHR) in the neonatal porcine ovary and its regulation by flutamide. Reprod. Domest. Anim. 46:377-384.

Faria, T. S., F. B. Brasil, F. J. B. Sampaio, and C. F. Ramos. 2010. Effects of maternal undernutrition during lactation on estrogen and androgen receptor expressions in rat ovary at puberty. Nutrition 26:993-999.

Fiedler, S. D., M. Z. Carletti, X. Hong, and L. K. Christenson. 
2008. Hormonal regulation of microrna expression in periovulatory mouse mural granulosa cells. Biol. Reprod. 79:1030-1037.

Fire, A., S. Xu, M. K. Montgomery, S. A. Kostas, S. E. Driver, and C. C. Mello. 1998. Potent and specific genetic interference by double-stranded rna in caenorhabditis elegans. Nature 391(6669):806-811.

Fortune, J., R. Cushman, C. Wahl, and S. Kito. 2000. The primordial to primary follicle transition. Mol. Cell. Endocrinol. 163:53-60.

Godfrey, K. M., P. D. Gluckman, and M. A. Hanson. 2010. Developmental origins of metabolic disease: Life course and intergenerational perspectives. Trends Endocrin. Metab. 21: 199-205.

Grzesiak, M., K. Knapczyk-Stwora, M. Duda, and M. Slomczynska. 2012. Elevated level of 17ß-estradiol is associated with overexpression of FSHR, CYP19A1, and CTNNB1 genes in porcine ovarian follicles after prenatal and neonatal flutamide exposure. Theriogenology 78:2050-2060.

Ibáñez, L., N. Potau, A. Ferrer, F. Rodriguez-Hierro, M. V. Marcos, and F. de Zegher. 2002. Reduced ovulation rate in adolescent girls born small for gestational age. J. Clin. Endocrinol. Metab. 87:3391-3393.

Iwasa, T., T. Matsuzaki, M. Murakami, R. Kinouchi, G. Gereltsetseg, S. Yamamoto, A. Kuwahara, T. Yasui, and M. Irahara. 2011. Delayed puberty in prenatally glucocorticoid administered female rats occurs independently of the hypothalamic Kiss1-Kiss1R-GnRH system. Int. J. Dev. Neurosci. 29:183-188.

Jia Y., R. Cong, R. Li, X. Yang, Q. Sun, N. Parvizi, and R. Zhao. 2012. Maternal low-protein diet induces gender-dependent changes in epigenetic regulation of the glucose-6-phosphatase gene in newborn piglet liver. J. Nutr. 142:1659-1665.

Kertesz, M., N. Iovino, U. Unnerstall, U. Gaul, and E. Segal. 2007. The role of site accessibility in microrna target recognition. Nat. Genet. 39:1278-1284.

Kezele, P. and M. K. Skinner. 2003. Regulation of ovarian primordial follicle assembly and development by estrogen and progesterone: Endocrine model of follicle assembly. Endocrinology 144:3329-3337.

Liu X., J. Wang, R. Li, X. Yang, Q. Sun, E. Albrecht, and R. Zhao. 2011. Maternal dietary protein affects transcriptional regulation of myostatin gene distinctively at weaning and finishing stages in skeletal muscle of Meishan pigs. Epigenetics 6:899-907.

Meikle, D. and M. Westberg. 2001. Maternal nutrition and reproduction of daughters in wild house mice (Mus musculus). Reproduction 122:437-442.
Nilsson, E. E. and M. K. Skinner. 2003. Bone morphogenetic protein-4 acts as an ovarian follicle survival factor and promotes primordial follicle development. Biol. Reprod. 69: $1265-1272$

Rae, M. T., C. E. Kyle, D. W. Miller, A. J. Hammond, A. N. Brooks, and S. M. Rhind. 2002. The effects of undernutrition, in utero, on reproductive function in adult male and female sheep. Anim. Reprod. Sci. 72:63-71.

Rucker, E. B., P. Dierisseau, K. U. Wagner, L. Garrett, A. Wynshaw-Boris, J. A. Flaws, and L. Hennighausen. 2000. Bcl$\mathrm{x}$ and bax regulate mouse primordial germ cell survival and apoptosis during embryogenesis. Mol. Endocrinol. 14:10381052.

Sirotkin, A. V., D. Ovcharenko, R. Grossmann, M. Lauková, and M. Mlynček. 2009. Identification of micrornas controlling human ovarian cell steroidogenesis via a genome-scale screen. J. Cell. Physiol. 219:415-420.

Sloboda, D. M., M. Hickey, and R. Hart. 2011. Reproduction in females: The role of the early life environment. Hum. Reprod. Update. 17:210-227.

Sloboda, D. M., G. J. Howie, A. Pleasants, P. D. Gluckman, and M. H. Vickers. 2009. Pre- and postnatal nutritional histories influence reproductive maturation and ovarian function in the rat. Plos One 4(8):e6744.

Sun, R., L. Lei, L. Cheng, Z. F. Jin, S. J. Zu, Z. Y. Shan, Z. D. Wang, J. X. Zhang, and Z. H. Liu. 2010. Expression of GDF-9, BMP-15 and their receptors in mammalian ovary follicles. J. Mol. Histol. 41:325-332.

Sun, Y., Y. Lin, H. Li, J. Liu, X. Sheng and W. Zhang. 2012. 2, 5hexanedione induces human ovarian granulosa cell apoptosis through bcl-2, bax, and caspase- 3 signaling pathways. Arch. Toxicol. 86:205-215.

Tanwar, P. S., T. O'Shea, and J. R. McFarlane, 2008. In vivo evidence of role of bone morphogenetic protein- 4 in the mouse ovary. Anim. Reprod. Sci. 106:232-240.

Tománek, M. and E. Chronowska. 2006. Immunohistochemical localization of proliferating cell nuclear antigen (PCNA) in the pig ovary. Folia Histochem. Cytobiol. 44:269-274.

Torley, K. J., J. C. da Silveira, P. Smith, R. V. Anthony, D. Veeramachaneni, Q. A. Winger, and G. J. Bouma. 2011. Expression of mirnas in ovine fetal gonads: Potential role in gonadal differentiation. Reprod. Biol. Endocrinol. 9:2.

Xu, B., J. Hua, Y. Zhang, X. Jiang, H. Zhang, T. Ma, W. Zheng, R. Sun, W. Shen, J. Sha, H. J. Cooke, and Q. Shi. 2011. Proliferating cell nuclear antigen (PCNA) regulates primordial follicle assembly by promoting apoptosis of oocytes in fetal and neonatal mouse ovaries. Plos One. 6(1):e16046. 